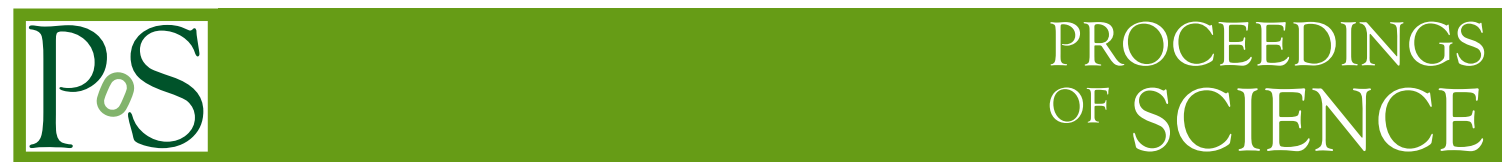

\title{
Non-neutralino Dark Matter
}

\section{Ki-Young CHOI $^{*}$}

Korea Astronomy and Space Science Institute

E-mail: kiyoungchoi@kasi.re.kr

Neutralino is a good candidate for dark matter. However there are more interesting and wellmotivated models of dark matter. In this talk I give a short review on some of the non-neutralino dark matter, especially about axion, axino, gravitino and right-handed scalar neutrino dark matter.

XXII. International Workshop on Deep-Inelastic Scattering and Related Subjects 28 April - 2 May 2014

Warsaw, Poland

${ }^{*}$ Speaker. 


\section{Introduction}

Dark matter (DM) is a solution to the missing mass problem to explain the discrepancies between the visible matter and the gravitational matter. The telescopes can directly observe matters which can absorb or emit light only. However the method using gravitational effects such as dynamics of the celestial body or the gravitational lensing can detect all forms of matter and energy, since the gravity is universal. The difference in both measurements exist in all scales from small scale to large scale of the Universe.

At least dark matter as a particle can explain all the observational anomalies in different scales, such as galaxy rotational curve, bullet cluster, large scale structure formation, cosmic microwave background radiation (CMB) and etc, with additional single component of particle. The properties of dark matter as a particle should satisfy

- Stability : DM have existed from early Universe as well as in the present Universe around galaxies and clusters.

- Neutrality : DM do not have electric charge and only the upper bound is given to the magnitudes of self interactions.

- Relic density : The amount of relic DM at present should be around 25\% of the energy contents of the present Universe.

- Coldness (or warm) : DM is non-relativistic to seed the structure formation properly.

There are many well-motivated candidates of dark matter such as WIMP, axion, axino, gravitino, sterile neutrino etc. The mass and interactions of the DM candidates spans over more than $10^{30}$ in the magnitude: mass from $\mu \mathrm{eV}$ to Planck mass $M_{P}$ and cross section from $1 / M_{P}^{2}$ to $1 / M_{Z}^{2}$. However the production of DM is model dependent and there are several different ways suggested up to now in the Literature

\section{Production of dark matter}

Dark matter should have existed from the early Universe at least before the relevant scales for the large scale structure formation enter the horizon, which might be just after big bang nucleosynthesis (BBN) epoch. Therefore DM production must have been completed before BBN. So the strong constraints are applied for the models where the production of DM occurs after this period.

Weakly interacting massive particle (WIMP) is one of the most popular candidate for DM. They were in the thermal equilibrium with thermal plasms in the early Universe and then decouples from them after it became non-relativistc. Therefore the number density is quite suppressed compared to the background radiation and it depends only on the annihilation cross section of DMs erasing all the information during the thermal equilibrium. Even though the number density is quite suppressed compared to the radiation, the energy density becomes enough to explain the relic density of DM due to the large mass of DM, which is typically around $100 \mathrm{GeV}$. The candidates of WIMPs include neutralino, scalar neutrino, RH neutrino, minimal DM, Klauza-Klein DM and so on. 
If the DM is charged particle, then there could be an asymmetry between DM particle and DM antiparticle. This asymmetry may affect the thermal decoupling of DM and thus the relic density changes depending on the asymmetry. The asymmetric dark matter has been tried to connect the DM asymmetry to that of baryon. Because with the similar number of asymmetry, the light DM of around $10 \mathrm{GeV}$ mass gives correct relic density for DM, they were used to explain the anomalies in the direct detection together.

The extremely weakly interacting massive particle (E-WIMP) may not be in the thermal equilibrium in the early Universe if the reheating temperature after inflation is not high enough. In that case, even though the interaction is not enough to make them equilibrium, the thermal plasma still can produce enough particles of DM, while the opposite process (E-WIMPs into thermal particles) is negligible since the number density of them is quite suppressed. This small amount of E-WIMPs can be enough to explain the relic density of DM if their mass is around $100 \mathrm{GeV}$. We will get to these examples such as gravitino and axino more in the next section.

For scalars, the classical value can be displaced from the vacuum in the early Universe. In this case, when the mass is comparable to the Hubble expansion, it begins to run from the misaligned position and then oscillate around the vacuum. The oscillating scalar behaves like non-relativistic matter and becomes a good candidate for DM. The famous example is axion, which has potential below the QCD phase transition and has coherent oscillations.

\section{Gravitino dark matter}

In supersymmetric theory, the lightest supersymmetric particle (LSP) is stable if the R-parity is conserved. The lightest neutralino is one example of WIMP DM and gravitino is another candidate for E-WIMP DM if it is LSP. The gravitino is the superpartner of graviton and the mass is generated when the SUSY is broken. The mass can be from $\mathrm{eV}$ to $\mathrm{TeV}$ depending on the supersymmetry breaking mechanism. The interaction is set from the theory itself, and is suppressed by Planck scale.

Since the interaction is very weak, the gravitinos could not be in the thermal equilibrium in the early Universe. However small amount could be produced from the thermal backgrounds by scatterings. This thermal production depends on the reheating temperature, $T_{\text {reh }}$, and the correct amount of DM can be produced if the reheating temperature around $10^{10} \mathrm{GeV}$ for the DM mass around $100 \mathrm{GeV}$. Therefore if the gravitino is the lightest supersymmetric particle and R-parity is preserved, it can be a good candidate for DM.

However due to the small interaction, it has serious constraints from the big bang nucleosynthesis and cosmic microwave background radiation. Because the next lightest supersymmetric particle can decay to Gravitino at late times usually after big bang nucleosynthesis epoch, the injection of high energetic particles into the plasma can destroy the standard production of light nuclei and change their abundance $[3,4,5]$. Or the large injection of energy can also change the spectrum of cosmic background radiation and make it deviate from Planckian distribution by observation. Therefore BBN and CMB put strong constraints on the next LSP and the production of the gravitino dark matter. Due to the BBN constraints, for GeV Gravitino DM, the neutralino NLSP is almost ruled-out, while stau NLSP is viable when the reheating temperature is less than around $10^{8} \mathrm{GeV}[1,2]$. 
When R-parity is broken, there is no more problem with BBN, since the NLSP decay much earlier than BBN epoch. Through the R-parity breaking interactions, Gravitino can decay with lifetime much longer than the age of the Universe. The decay of Graviitnos can produce gammarays that can be detectable in the future Gamma-ray observation $[6,7,8]$.

\section{Axino dark matter}

The axino is the fermionic superpartner of the axion, which is introduced to explain the strong $\mathrm{CP}$ problem. The interaction of axino to ordinary matter is determined by the Peccei-Quinn scale, which is constrained to be between $10^{9} \mathrm{GeV}$ and $10^{12} \mathrm{GeV}$ from the energy loss in the stars and overabundance of axion production. The mass of axino is also model dependent and considered to be between $\mathrm{keV}$ and Gravitino mass.

There are two representative models of axion, KSVZ model [9] and DFSZ model [10]. For KSVZ model, the axion interaction is mediated by the heavy quarks and the axinos are produced from the scatterings or decays of the thermal particles. The axino abundance from scatterings is proportional to the reheating temperature, while those from the decays of thermal particles such as gluino, squark or neutralinos is independent on the reheating temperature once the temperature is large enough to produce the heavy thermal particles. For DFSZ model, axino production is dominated by the Higgsino coupling, which is also temperature independent $[11,12,13]$.

There is also non-thermal production of axinos from the decay of NLSP after out-of-equilibrium. For heavy axinos with mass larger than around $100 \mathrm{MeV}$, the non-thermal production can be dominant source for axino production. For light axinos, then the thermal production can be the main source for the production of axino dark matter [14, 15, 16].

If axino is heavy, then it can decay to produce DM $[17,18]$. The large production of entropy from the decay of axinos can dilute the gravitinos and thus relaxes the problem of gravitino. In this case, a large annihilation cross section of neutralino is needed, and thus Higgsino-like neutralino is preferred.

\section{Right-handed scalar neutrino dark matter}

In a supersymmetric neutrinophilic Higgs model, the right-handed sneutrino can be in the thermal equilibrium in the early Universe with the large Yukawa coupling and small vacuum expectation value in the neutrinophilic Higgs model [20, 21]. However the large Yukawa coupling generates a lot of problems together such as lepton flavour violation, collider constraints from LHC and LEP, muon decay experiments and dark matter relic density. However there could be a small region where the deviation of the predicted muon anomalous magnetic moment in the standard model from the observation can be explained in this model [22].

\section{Discussion}

Dark matter is one of the fundamental problems in the cosmology, astrophysics and particle physics. The existence is evident from many observations and only the identification is left at the moment. For WIMP, the interaction of DM might be the weak scale and fortunately detected in 
the direct, indirect detection or in the collider. For E-WIMP such as axion, axino, and gravitino, even though the interaction is very weak, the correct abundance of DM can be obtained. Their detection might be more difficult and depends on the model. Axions can be detected in the solar axion experiment or in the cavity experiment. However the gravitino or axino is quite difficult to probe in the direct detection. However it might give signatures in the collider as missing energy or in the astrophysical signatures.

\section{References}

[1] D. G. Cerdeno, K. Y. Choi, K. Jedamzik, L. Roszkowski and R. Ruiz de Austri, JCAP 0606 (2006) 005 [arXiv:hep-ph/0509275].

[2] S. Bailly, K. -Y. Choi, K. Jedamzik and L. Roszkowski, JHEP 0905 (2009) 103 [arXiv:0903.3974 [hep-ph]].

[3] K. Jedamzik, Phys. Rev. D 70 (2004) 063524 [arXiv:astro-ph/0402344].

[4] M. Kawasaki, K. Kohri and T. Moroi, Phys. Lett. B 625 (2005) 7 [astro-ph/0402490].

[5] M. Kawasaki, K. Kohri, T. Moroi and A. Yotsuyanagi, Phys. Rev. D 78 (2008) 065011 [arXiv:0804.3745 [hep-ph]].

[6] Ki-Young Choi, D. E. Lopez-Fogliani, C. Munoz and R. R. de Austri, JCAP 1003 (2010) 028 [arXiv:0906.3681 [hep-ph]].

[7] Ki-Young Choi and C. E. Yaguna, Phys. Rev. D 82 (2010) 015008 [arXiv:1003.3401 [hep-ph]].

[8] Ki-Young Choi, D. Restrepo, C. E. Yaguna and O. Zapata, JCAP 1010 (2010) 033 [arXiv:1007.1728 [hep-ph]].

[9] J. E. Kim, Phys. Rev. Lett. 43 (1979) 103; M. A. Shifman, V. I. Vainstein, V. I. Zakharov, Nucl. Phys. B 166 (1980) 4933.

[10] M. Dine, W. Fischler and M. Srednicki, Phys. Lett. B 104 (1981) 199; A. P. Zhitnitskii, Sov. J. Nucl. Phys. 31260 (1980).

[11] E. J. Chun, Phys. Rev. D84 043509 (2011) [arXiv:1104.2219 [hep-ph]].

[12] K. J. Bae, K. Choi and S. H. Im, JHEP 1108 (2011) 065 [arXiv:1106.2452 [hep-ph]].

[13] K. J. Bae, E. J. Chun and S. H. Im, JCAP 1203 (2012) 013 [arXiv:1111.5962 [hep-ph]].

[14] L. Covi, J. E. Kim and L. Roszkowski, Phys. Rev. Lett. 82 (1999) 4180 [hep-ph/9905212].

[15] L. Covi, H. B. Kim, J. E. Kim and L. Roszkowski, JHEP 0105 (2001) 033 [hep-ph/0101009].

[16] K. -Y. Choi, L. Covi, J. E. Kim and L. Roszkowski, JHEP 1204 (2012) 106 [arXiv:1108.2282 [hep-ph]].

[17] Ki-Young Choi, Jihn E. Kim, H. M. Lee, and O. Seto, Phys. Rev. D 77 (2008) 123501 [arXiv: 0801.0491[hep-ph]].

[18] Kiwoon Choi, K. -Y. Choi and C. S. Shin, Phys. Rev. D 86 (2012) 083529 [arXiv:1208.2496 [hep-ph]].

[19] Ki-Young Choi, Jihn E. Kim and L. Roszkowski, J. Korean Phys. Soc. 63 (2013) 1685 [arXiv:1307.3330 [astro-ph.CO]]. 
[20] K. -Y. Choi and O. Seto, Phys. Rev. D 86043515 (2012) [Erratum-ibid. D 86089904 (2012)]. [arXiv:1205.3276 [hep-ph]].

[21] K. -Y. Choi and O. Seto, Phys. Rev. D 88 (2013) 035005 [arXiv:1305.4322 [hep-ph]].

[22] K. -Y. Choi, O. Seto and C. S. Shin, arXiv:1406.0228 [hep-ph]. 